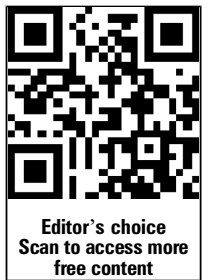

- Additional material is published online only. To view please visit the journal online (http://dx.doi.org/10.1136/ bjsports-2014-093500).

${ }^{1}$ Department of Family Medicine, University of Tennessee, Knoxville, Tennessee, USA ${ }^{2}$ Department of Family Medicine, University of Washington, Seattle, Washington, USA ${ }^{3}$ Division of Cardiology, Department of Pediatrics, University of Washington, Seattle, Washington, USA

\section{Correspondence to} Irfan M Asif, Department of Family Medicine, University of Tennessee 1927 Alcoa Hwy U-67, Knoxville, TN 37920, USA; iasif@utmck.edu

Accepted 19 April 2014 Published Online First 13 May 2014

\title{
The psychological impact of cardiovascular screening: the athlete's perspective
}

\author{
Irfan M Asif, ${ }^{1}$ Serena Johnson, ${ }^{2}$ Jason Schmieg, ${ }^{1}$ Tiffany Smith, ${ }^{1}$ Ashwin L Rao, ${ }^{2}$ \\ Kimberly G Harmon, ${ }^{2}$ Jack C Salerno, ${ }^{3}$ Jonathan A Drezner ${ }^{2}$
}

\begin{abstract}
Background Published guidelines suggest that ECG screening in US athletes may cause excessive anxiety, especially in those with false-positive findings. However, this has never been formally evaluated.

Methods and study design Prospective, nonrandomised controlled trial. High school athletes received a standardised history and physical examination (control) or a history and physical examination with an ECG (experimental). Prescreen and postscreen assessments for health attitudes, anxiety and impact of screening on sport were conducted.
\end{abstract}

Results 952 athletes $(49.7 \%$ girls, mean age 15.5 years) participated (control $=150$; experimental=802). $4.4 \%$ worried about having an underlying cardiac condition, and $73 \%$ wanted to learn if they had a cardiac abnormality prior to competition. In the experimental group, 576 had normal screens, 220 had an abnormal screen (by history 15.8\%, physical examination $6.2 \%$ or ECG $1.7 \%$ ) but normal work up (false-positive) and 6 were identified with a serious cardiac condition (true-positive, $0.75 \%$ ). Compared with the control group, those who received an ECG were more likely to: (1) be significantly more satisfied with their screening $(p<0.001)$, (2) feel safer during competition $(p<0.01)$, (3) support that all athletes should receive cardiac screening $(p<0.001)$ and $(4)$ state the ECG positively impacted their training $(p<0.001)$.

False-positive athletes did not report anxiety during or after screening. Distress levels did not differ based on reason for needing further evaluation (history, physical examination or $E C G, p=0.311)$. Compared with control participants, individuals with false-positive results: (1) reported no difference in postscreen anxiety $(p=0.775)$, (2) felt safer during competition $(p<0.001)$, (3) would recommend ECG screening to others $(p<0.001)$ and $(4)$ expressed a positive impact on training $(p<0.001)$.

Conclusions Excessive anxiety should not be used as a reason to forego ECG screening in athletes.

\section{INTRODUCTION}

Sudden cardiac death (SCD) is the leading cause of death during exercise. ${ }^{1}$ There is international debate regarding the most effective screening protocol for young competitive athletes. ${ }^{2-8}$ The European Society of Cardiology (ESC), FIFA, IOC and many professional sporting organisations recommend a preparticipation screening evaluation that includes a resting ECG in addition to a history and physical examination. ${ }^{9-11}$ The American Heart Association (AHA) recommends only an evaluation with a history and physical examination, citing several concerns with ECG screening. ${ }^{12}$ This includes the potential for excessive anxiety in athletes who receive an ECG, especially those with false-positive (FP) findings. ${ }^{12}$ A working group of the National Heart, Lung, and Blood Institute (NHLBI) has additionally outlined a need to understand the psychological impact of cardiovascular screening in athletes prior to widespread implementation of new cardiac screening protocols. ${ }^{13}$ To date, distress associated with ECG screening has never been investigated in US athletes. The purpose of this study was to determine the psychological implications of cardiovascular screening in athletes, with and without utilisation of an ECG.

\section{METHODS}

This was a prospective, non-randomised controlled trial where athlete's were screened based on their school's normal screening protocol (eg, history and physical examination vs history and physical examination+ECG). The study consisted of two groups (experimental=ECG group and control=no ECG group) who received a similar three-phase protocol: (1) prescreen psychological assessment, (2) cardiovascular screen and (3) postscreen psychological evaluation.

The ECG group consisted of athletes who received a history and physical examination and ECG. The cardiovascular screen was based on the AHA 12-element history and physical examination, followed by an ECG that was interpreted using the 2013 Seattle Criteria. ${ }^{14}$ If any element of the history and physical examination or ECG was found to be abnormal, the athlete was referred for further cardiac testing. On-site echocardiograms were provided for all athletes with concerning findings within their history, physical examination or ECG. All cardiac testing was completed prior to undergoing the postscreen psychological evaluation.

The control group consisted of 150 consecutively screened age-matched and gender-matched athletes who had a normal evaluation and were cleared to play with the traditional AHA-based history and physical examination, with no ECG or further cardiac evaluation.

Prescreen and postscreen psychological assessments were given to both groups. The prescreen assessment consisted of questions evaluating baseline measures of anxiety, health attitudes, concern for heart disease, attitudes regarding the potential for further testing and interest in cardiac screening prior to sports participation (see online supplementary file 1). Baseline screening for trait anxiety was assessed using the validated anxiety subsection of the Primary Care Evaluation of Medical Disorders (PRIME-MD). ${ }^{15}$ 
The postscreen assessment was adapted from Solberg et al, ${ }^{16}$ who measured distress in Norwegian soccer players following cardiovascular screening (see online supplementary files 2-4). The postscreen instrument was given after completion of all cardiac testing and contained questions evaluating satisfaction with screening, feelings of anxiety during and immediately after screening, belief about whether other athletes should receive their particular cardiac screen prior to competition and impact of cardiac screening on the individual's athletic activity. A fivepoint Likert scale $(-2=$ strongly disagree, $-1=$ disagree, $0=$ neutral, $1=$ agree and $2=$ strongly agree) was used to measure athlete preferences during the prescreen and postscreen psychological evaluations. Mean scores and SD were reported.

The tools used in this study were validated prior to their use. The reliability using Cronbach's $\alpha$ was found to be $0.73-0.82$. Student $\mathrm{t}$ tests and one-way between-subjects analyses of variance (ANOVA) with Tukey follow-up tests were run to determine differences between groups. Statistical analyses were performed on differences between: (1) experimental and control groups, (2) control group and the subgroups of normal-screened (athletes cleared without further testing), FP and true-positive individuals in the experimental group and (3) reason for FP test (history, physical examination or ECG). A $\mathrm{p}<0.05$ was considered significant. Any clinically relevant cardiac abnormality including disorders associated with SCD as well as minor conditions requiring medical follow-up, surveillance or management were considered true-positives.

The study was approved by the Human Subjects Division at the University of Tennessee Graduate School of Medicine.

\section{RESULTS}

A total of 952 high school student-athletes (49.7\% girls, average age 15.5 years) participated in the study (ECG group $=802$ and no ECG group $=150$ (figure 1$)$ ). Of all participants, $4.4 \%$ worried about having an underlying cardiac condition, and 73\% preferred to know if they had a cardiovascular abnormality prior to competition. Athletes participated in a variety of sports with the most common being basketball, football, soccer and track (figure 2).

Baseline characteristics are given in table 1. Athletes receiving an ECG were more worried about having an underlying heart condition compared with athletes who did not receive an ECG $(5.0 \%$ vs $1.4 \%, p<0.001)$. Based on screening from the PRIME-MD, the trait of anxiety was more pronounced in the ECG group compared with the control group (48.6\% vs $27.0 \%$, $\mathrm{p}<0.001)$. A portion of athletes in both groups reported having a family member or close friend who had died at an early age of $<50$ years (no ECG group $12.2 \%$ vs ECG group 16.2\%). Ongoing medical problems were more common in the ECG group $(18.5 \%)$ versus the no ECG group $(9.3 \%$; $<<0.001)$.

In the experimental group, 576 athletes screened normal with no need for further cardiac testing. Two hundred and twenty athletes had an abnormal screen (by history 15.8\%, physical examination $6.2 \%$ or ECG $1.7 \%$ ) but normal subsequent work up (FP). Six athletes were identified with a serious cardiovascular abnormality (true-positive, $0.75 \%$ ).

\section{ECG group versus no ECG group}

Neither the control nor experimental groups reported anxiety during cardiovascular screening (control group mean $=-0.8$ $(\mathrm{SD}=1.02)$, experimental group mean $=-0.22 \quad(\mathrm{SD}=1.10)$, $\mathrm{p}<0.001$, figure 3 ) and there was no significant difference in distress levels immediately after screening (control group mean $=-0.99, \quad \mathrm{SD}=0.94, \quad$ experimental group mean $=-0.85$, $\mathrm{SD}=0.91, \mathrm{p}=0.88)$. Those who received an ECG: (1) were more satisfied with screening $(p<0.001)$, (2) felt safer during competition $(\mathrm{p}<0.01)$, (3) were more supportive of
Figure 1 Study flow diagram (H\&P, history and physical examination; HS, high school).

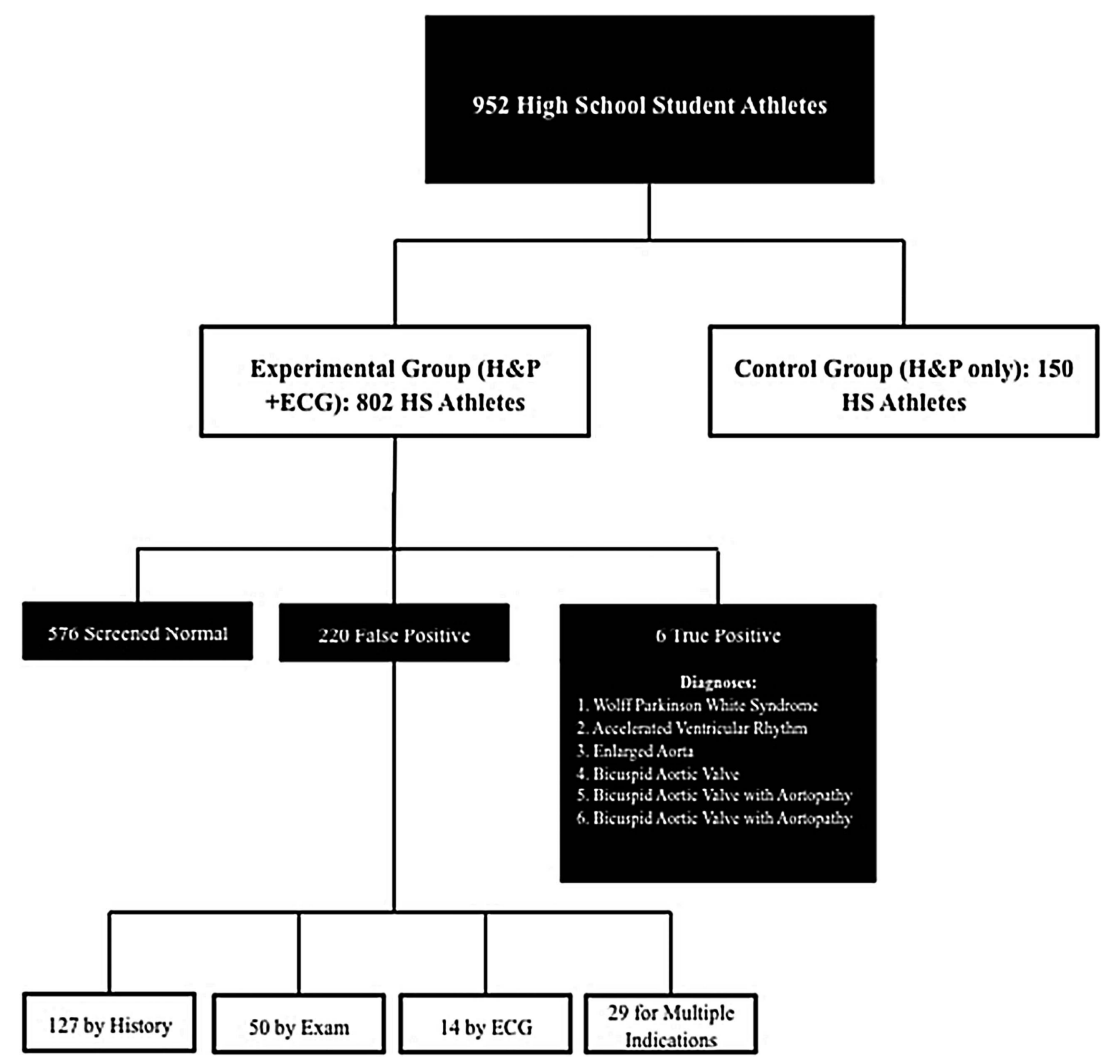


Figure 2 Sports participation among the ECG and no ECG group.
Percentage of Sports Participation Among High School Athletes

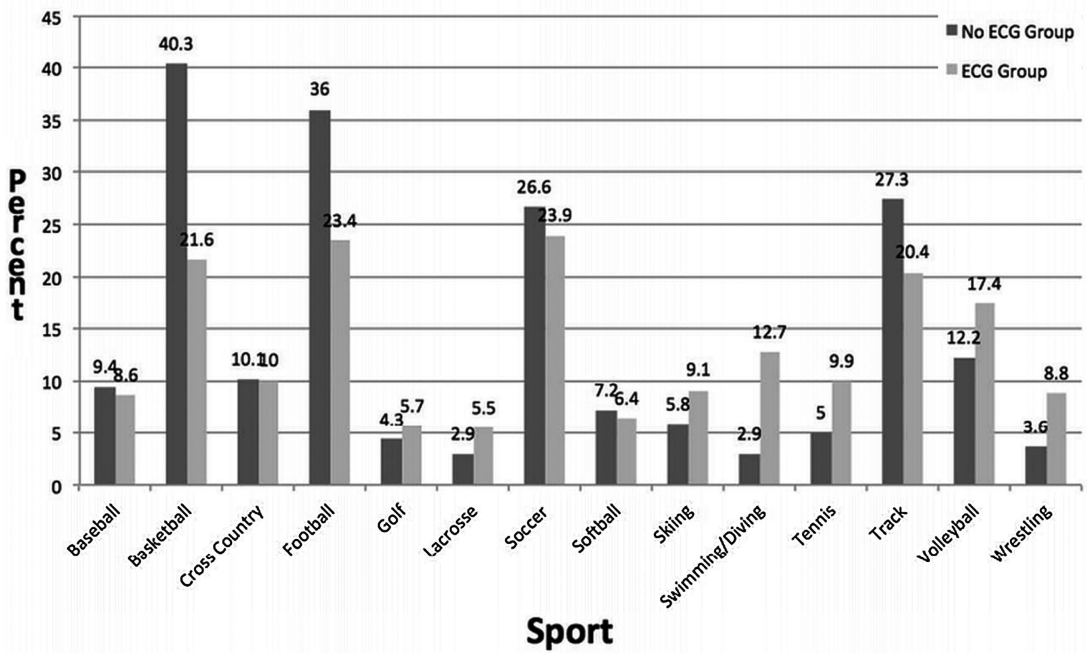

cardiovascular screening in athletes $(p<0.001)$ and $(4)$ stated the ECG positively impacted their training $(\mathrm{p}<0.001)$.

\section{Experimental subgroup analysis}

When the ECG group was subdivided by screening result (normal, FP or true-positive), normal and FP athlete subgroups did not report anxiety during (normal $=-0.23, \mathrm{SD}=1.04 ; \mathrm{FP}=$ $-0.21, \mathrm{SD}=1.24$ ) or after screening (normal $=-0.82, \mathrm{SD}=0.86$; $\mathrm{FP}=-0.94, \mathrm{SD}=1.06$ ) with no significant difference between these groups at either time period (anxiety during $p=0.996$, anxiety after $\mathrm{p}=0.207$; figure 4 ).

Compared with the no ECG group, athletes who screened normal in the ECG group reported being more satisfied with their cardiac screen $(p<0.001)$, felt that all athletes should receive their cardiac screening protocol $(p<0.001)$ and expressed a more positive impact on their training $(p<0.001$; figure 4).

When compared with the no ECG group, individuals with FP results: (1) reported no difference in postscreen anxiety $(p=0.775)$, (2) felt safer during competition $(p<0.001)$, (3) would recommend ECG screening to others $(p<0.001)$ and $(4)$ expressed a positive impact on training $(p<0.001$; figure 4$)$. In those who received a FP result, distress levels did not differ based on the reason for needing further evaluation (history, physical examination or ECG, $\mathrm{p}=0.311$ (figure 5)). It appeared that the group of FP resulting from an abnormal ECG had the least amount of anxiety (more strongly disagreed on the Likert scale) than the athletes with a FP from a history or physical examination; however, this difference was not statistically significant. FP athletes did not report feeling as if they were more likely to develop a future cardiac condition compared with their peers (mean $=-0.6, \mathrm{SD}=1.00$ ).

Athletes who were found to have an underlying cardiac disorder (true-positive) did describe anxiety during (mean $=0.5, \mathrm{SD}=1.05$ ) and after (mean $=0.14, \mathrm{SD}=1.47$ ) screening (figure 4$)$. These athletes were restricted from sports until further work up and/or treatment was appropriately performed by their treating cardiologist. They were satisfied with their cardiac screening (mean $=0.67$, $\mathrm{SD}=1.03$ ) and would recommend ECG screening to others $($ mean $=1.5, \mathrm{SD}=0.55$; figure 5$)$.

\section{DISCUSSION}

The role of ECG in screening athletes for occult cardiovascular disease is controversial. Recent investigations have begun to address concerns regarding FP rates, cost-effectiveness and infrastructure development. ${ }^{7}{ }^{17-29}$ The current study is the first large-scale investigation into the psychological impact of ECG screening in young competitive high school athletes and provides important perspectives and preferences from the athletes who actually undergo the screening process.

Table 1 Baseline characteristics

\begin{tabular}{lll}
\hline & No ECG group & ECG group \\
\hline Participants & $\mathrm{N}=150$ & $\mathrm{~N}=802$ \\
Male (\%) & 49 & 51 \\
Female (\%) & 51 & 49 \\
Age (years old) & 15.5 & 15.5 \\
Ethnicity & $91 \%$ Caucasian, & $71.2 \%$ Caucasian, $10.6 \%$ Asian, \\
& $4.7 \%$ African-American, 2.7\% mixed & $9.5 \%$ mixed, 3.9\% African-American, \\
Ongoing medical conditions (\%) & $9.3(6$ asthma) & $3 \%$ Hispanic \\
Positive response to the PRIME-MD screening for possible trait anxiety (\%) & 27 & $18.5(10$ asthma) \\
Family member or close friend who has died of cardiac disease <50 years old (\%) & 12.20 & 48.60 \\
Worried about having a heart condition (\%) & 1.40 & 16.20 \\
\hline
\end{tabular}

PRIME-MD, Primary Care Evaluation of Medical Disorders. 


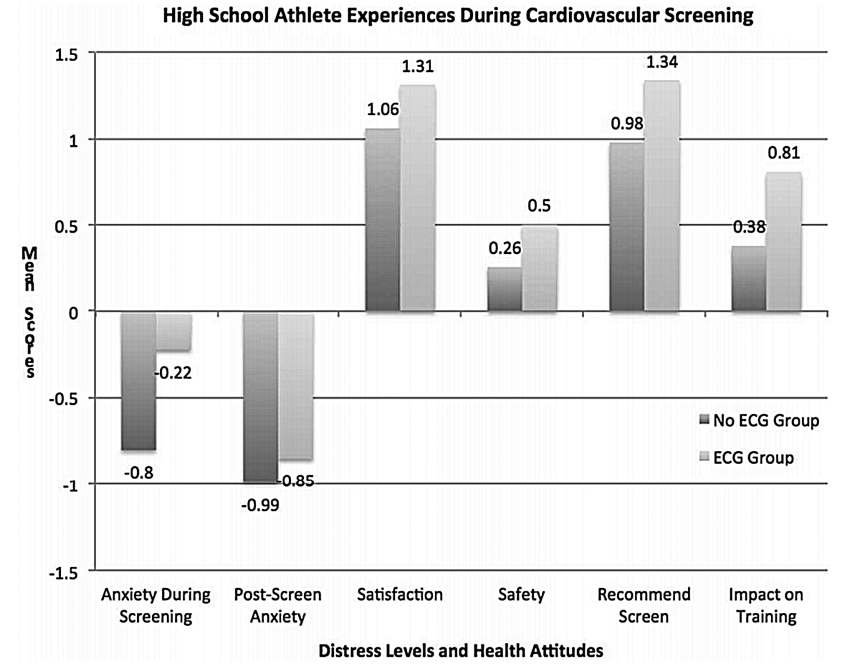

Figure 3 No measurable distress reported in either group during screening. Responses were graded on a five-point Likert scale ( $-2=$ strongly disagree to $2=$ strongly agree).

The 2007 AHA statement on cardiovascular screening states that FP results from an ECG screening programme would lead to unnecessary life implications, emotional burdens and unnecessary anxiety. ${ }^{12}$ The results from the present investigation demonstrate that ECG screening does not cause excessive anxiety in athletes, including those who screen FP. Importantly, there were also no differences in distress levels based on the reason for a FP evaluation (ie, history, physical examination or ECG). Athletes also reported numerous psychological benefits that previously have not been considered when discussing the psychological impact of ECG screening. Based on the results in this study, psychological distress should not be used as a rationale to forego ECG screening.

Athletes who were found to have a cardiac disorder did describe more anxiety, yet were still satisfied with their evaluation and would recommend ECG screening to other athletes. A larger sample is needed to further investigate the psychological implications of being detected with a cardiac disorder.
Unlike the majority of FP diagnoses, a true-positive finding can deliver a significant psychological burden, regardless of the cardiovascular screening method. Athletes often consider themselves to be among the healthiest segment of society and believe they are unlikely to have an underlying medical condition. This is consistent with findings from this study, which demonstrated that $<5 \%$ of athletes were concerned about having an underlying condition prior to cardiovascular screening. As expected, athletes who are diagnosed with a serious cardiac disorder may be in denial, shock or disbelief when first learning about their condition. Additionally, athletes with a potentially lethal cardiac disorder (found regardless of the screening protocol) may be disqualified from competition, which could have a profound impact on their identity and additional psychological implications. Cardiovascular screening programmes should consider developing support mechanisms for these individuals given the emotional consequences that may occur after diagnosis.

\section{LIMITATIONS}

Despite the large sample size found in this study, there was limited racial and ethnic diversity and a small number of truepositive findings. Further investigations are needed to fully evaluate the psychological impact of screening based on racial and ethnic diversity as there may be important differences within these subgroups. In addition more studies are needed to investigate the impact of true-positive results.

This was a non-randomised study, which could introduce selection bias. More studies need to be performed in a randomised manner to better characterise the findings. While the two groups were age and gender matched, there was a 5:1 ratio in the sample of experimental to control group athletes, which may make the results less generalisable. This yielded slight differences in baseline perspectives (eg, 1.4\% of athletes in the control groups worried about having an underlying cardiac condition compared with $5 \%$ in the experimental group). However, these differences did not appear to be significant since the experimental group did not report excessive distress compared with the control group. We also were not able to directly compare subgroups from the experimental group with the control group (ie, FP from the experimental group vs FP from
Figure 4 Athletes diagnosed with disease reported distress during and after screening, while other subgroups described no measurable anxiety. Responses were graded on a five-point Likert scale $(-2=$ strongly disagree to $2=$ strongly agree).
Psychological Impact of Cardiovascular Screening Based Screening Outcome

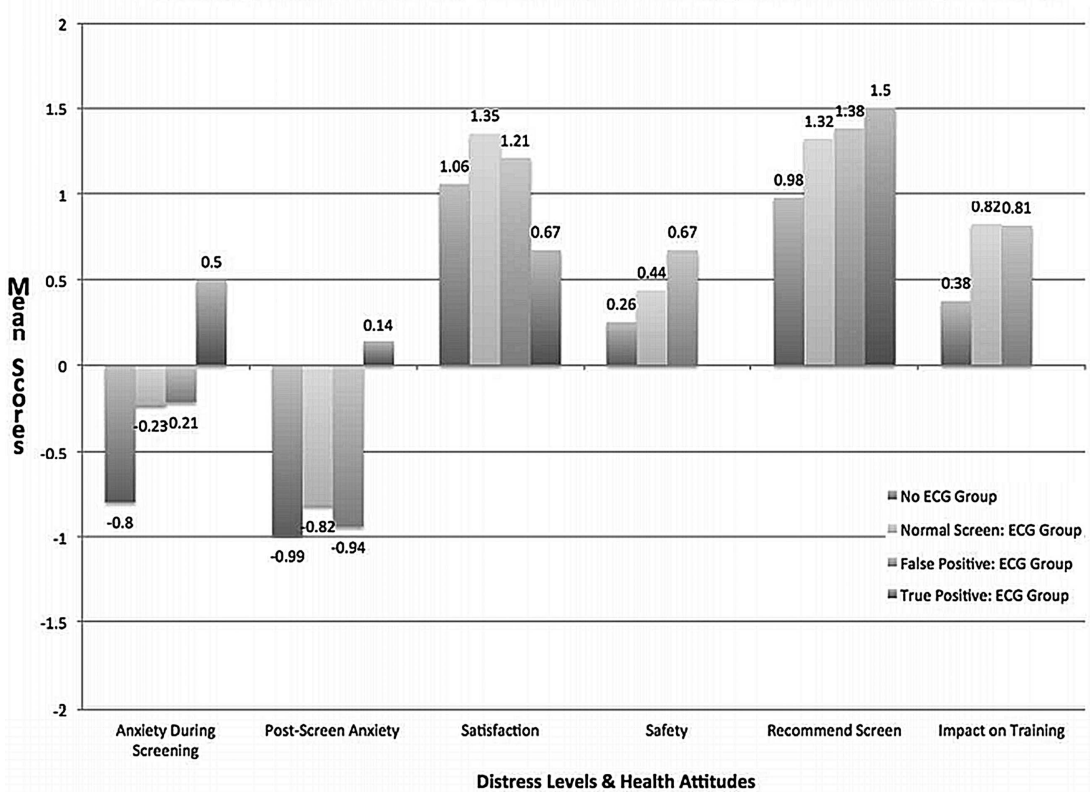


No Perceptible Anxiety Found During Screening When Segregated by Reason for False Positive Result

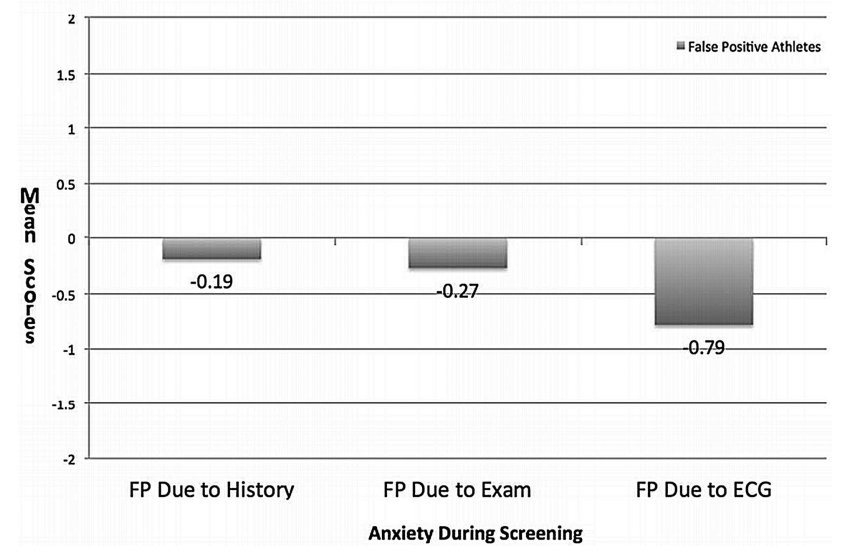

Figure 5 There was no statistically significant difference in anxiety levels during screening for false-positive (FP) athletes requiring further work up based on history, examination or ECG. Responses were graded on a five-point Likert scale ( $-2=$ strongly disagree to $2=$ strongly agree).

the control group). However, since anxiety levels did not differ based on the reason for FP result (history, physical examination or ECG) in our experimental group, one may infer that there would be no difference in distress levels between athletes with FP results regardless of the reason for additional testing.

Data was also obtained in high school student-athletes only and may not be generalisable to the college or professional setting. Future studies should consider investigating the psychological impact of cardiovascular screening in these populations. Finally, this study utilised on-site echocardiography to evaluate the abnormal screening findings. There may be differences in psychological implications for athletes who cannot undergo same day follow-up evaluation. However, based on findings from this study, the psychological impact of a delayed work up of abnormal findings should be independent of the indication for further evaluation (ie, abnormal history, physical examination or ECG).

\section{CONCLUSION}

Sudden cardiac arrest and death in sport remains a major public health concern. Medical communities and athletic programmes have debated the optimal cardiovascular preparticipation screening protocol, but often lost in the discussion are the perspectives and preferences of the athletes themselves. This is the first prospective study investigating the psychological impact of cardiovascular screening in US high school athletes and results from

\section{What are the new findings?}

- No study has examined the psychological impact of ECG screening in US athletes.

- ECG screening does not cause undue anxiety in US high school athletes, even in those with false-positive results. Thus, it should not be a reason to forego advanced cardiovascular screening with an ECG.

- There are a number of positive psychological benefits of ECG screening that are often not discussed when delineating the impact of advanced screening modalities. this large-scale investigation demonstrate that ECG screening does not cause excessive anxiety in this population. Consequently, undue distress should not be used as an argument to forego advanced preparticipation cardiovascular screening strategies inclusive of ECG.

Contributors IMA was responsible for design, data collection, analysis and manuscript preparation. SJ and IS were responsible for data collection and entry. TS was responsible for statistical analysis and manuscript review. ALR, KGH and JCS were responsible for clinical assessment of athletes and manuscript review. JAD was responsible for the study design, athlete care and manuscript review.

\section{Competing interests None.}

Provenance and peer review Not commissioned; externally peer reviewed.

\section{REFERENCES}

1 Harmon KG, Asif IM, Klossner D, et al. Incidence of sudden cardiac death in national collegiate athletic association athletes. Circulation 2011;123:1594-600.

2 Sharma S, Estes NA, Vetter VL, et al. Clinical decisions. Cardiac screening before participation in sports. N Engl J Med 2013;369:2049-53.

3 Sharma S. Point/Mandatory ECG screening of young competitive athletes. Heart Rhythm 2012;9:1642-5.

4 Sharma S. Point/Mandatory ECG screening of young competitive athletes. Heart Rhythm 2012;9:1896.

5 Maron BJ. Counterpoint: mandatory ECG screening of young competitive athletes. Heart Rhythm 2012;9:1646-9.

6 Maron BJ. Counterpoint/mandatory ECG screening of young competitive athletes. Heart Rhythm 2012;9:1897.

7 Asif IM, Drezner JA. Sudden cardiac death and preparticipation screening: the debate continues-in support of electrocardiogram-inclusive preparticipation screening. Prog Cardiovasc Dis 2012;54:445-50.

8 Estes NA, Link MS. Preparticipation athletic screening including an electrocardiogram: an unproven strategy for prevention of sudden cardiac death in the athlete. Prog Cardiovasc Dis 2012;54:451-4.

9 Ljungqvist A, Jenoure PJ, Engebretsen L, et al. The International Olympic Committee (IOC) consensus statement on periodic health evaluation of elite athletes, March 2009. Clin J Sport Med 2009;19:347-65.

10 Dvorak J, Grimm K, Schmied C, et al. Development and implementation of a standardized precompetition medical assessment of international elite football players-2006 FIFA World Cup Germany. Clin J Sport Med 2009;19:316-21.

11 Corrado D, Pelliccia A, Bjørnstad HH, et al. Cardiovascular pre-participation screening of young competitive athletes for prevention of sudden death: proposal for a common European protocol. Consensus statement of the study group of sport cardiology of the working group of cardiac rehabilitation and exercise physiology and the working group of myocardial and pericardial diseases of the European Society of Cardiology. Eur Heart J 2005;26:516-24.

12 Maron BJ, Thompson PD, Ackerman MJ, et al. Recommendations and considerations related to preparticipation screening for cardiovascular abnormalities in competitive athletes: 2007 update: a scientific statement from the American Heart Association Council on Nutrition, Physical Activity, and Metabolism: endorsed by the American College of Cardiology Foundation. Circulation 2007:115:1643-55

13 Kaltman JR, Thompson PD, Lantos J, et al. Screening for sudden cardiac death in the young: report from a national heart, lung and blood institute working group. Circulation 2011;123:1911-18

14 Drezner JA, Ackerman MJ, Anderson J, et al. Electrocardiographic interpretation in athletes: the 'Seattle criteria'. Br J Sports Med 2013;47:122-4.

15 Spitzer RL, Kroenke K, Williams JB. Validation and utility of a self-report version of PRIME-MD: the PHQ primary care study. Primary care evaluation of mental disorders. Patient Health Questionnaire. JAMA 1999:282:1737-44.

16 Solberg EE, Bjørnstad TH, Andersen TE, et al. Cardiovascular pre-participation screening does not distress professional football players. Eur J Prev Cardiol 2012;19:571-7

17 Marek J, Bufalino V, Davis J, et al. Feasibility and findings of large-scale electrocardiographic screening in young adults: data from 32,561 subjects. Heart Rhythm 2011;8:1555-9.

18 Weiner RB, Hutter AM, Wang F, et al. Performance of the 2010 European Society of Cardiology criteria for ECG interpretation in athletes. Heart 2011;97:1573-7.

19 Brosnan M, La Gerche A, Kalman J, et al. The Seattle criteria increase the specificity of preparticipation ECG screening among elite athletes. Br J Sports Med 2014:48:1144-50.

20 Drezner JA. ECG screening in athletes: time to develop infrastructure. Heart Rhythm 2011;8:1560-1.

21 Drezner JA, Asif IM, Owens DS, et al. Accuracy of ECG interpretation in competitive athletes: the impact of using standardised ECG criteria. Br J Sports Med 2012:46:335-40. 
22 Drezner JA, Fischbach $\mathrm{P}$, Froelicher $\mathrm{V}$, et al. Normal electrocardiographic findings: recognising physiological adaptations in athletes. Br J Sports Med 2013;47:125-36.

23 Drezner JA, Ashley E, Baggish AL, et al. Abnormal electrocardiographic findings in athletes: recognising changes suggestive of cardiomyopathy. Br I Sports Med 2013:47:137-52.

24 Drezner JA, Ackerman MJ, Cannon BC, et al. Abnormal electrocardiographic findings in athletes: recognising changes suggestive of primary electrical disease. $\mathrm{Br}$ J Sports Med 2013;47:153-67.

25 Price DE, McWilliams A, Asif IM, et al. Electrocardiography-inclusive screening strategies for the detection of cardiovascular abnormalities in high school athletes. Heart Rhythm 2014;11:442-9.
26 Asif IM, Rao AL, Drezner JA. Sudden cardiac death in young athletes: what is the role of screening? Curr Opin Cardiol 2013;28:55-62.

27 Leslie LK, Cohen JT, Newburger JW, et al. Costs and benefits of targeted screening for causes of sudden cardiac death in children and adolescents. Circulation 2012:125:2621-9.

28 Wheeler MT, Heidenreich PA, Froelicher VF, et al. Cost-effectiveness of preparticipation screening for prevention of sudden cardiac death in young athletes. Ann Intern Med 2010;152:276-86.

29 Schoenbaum M, Denchev P, Vitiello B, et al. Economic evaluation of strategies to reduce sudden cardiac death in young athletes. Pediatrics 2012;130: e380-9.

$\underline{\underline{w}}$ 